\title{
Characteristic evolutions in numerical relativity using six angular patches
}

\author{
Christian Reisswig ${ }^{1}$, Nigel T Bishop ${ }^{2}$, Chi Wai Lai ${ }^{2}$, \\ Jonathan Thornburg ${ }^{1}$ and Bela Szilagyi ${ }^{1}$ \\ ${ }^{1}$ Max-Planck-Institut für Gravitationsphysik, Albert-Einstein-Institut, Am Mühlenberg 1, \\ D-14476 Golm, Germany \\ 2 Department of Mathematical Sciences, University of South Africa, PO Box 392, Unisa 0003 , \\ South Africa
}

Received 5 October 2006, in final form 11 December 2006

Published 4 June 2007

Online at stacks.iop.org/CQG/24/S327

\begin{abstract}
The characteristic approach to numerical relativity is a useful tool in evolving gravitational systems. In the past this has been implemented using two patches of stereographic angular coordinates. In other applications, a six-patch angular coordinate system has proved effective. Here we investigate the use of a sixpatch system in characteristic numerical relativity, by comparing an existing two-patch implementation (using second-order finite differencing throughout) with a new six-patch implementation (using either second- or fourth-order finite differencing for the angular derivatives). We compare these different codes by monitoring the Einstein constraint equations, numerically evaluated independently from the evolution. We find that, compared to the (second-order) two-patch code at equivalent resolutions, the errors of the second-order sixpatch code are smaller by a factor of about 2 , and the errors of the fourth-order six-patch code are smaller by a factor of nearly 50 .
\end{abstract}

PACS number: $04.25 . \mathrm{Dm}$

M This article features online multimedia enhancements

(Some figures in this article are in colour only in the electronic version)

\section{Introduction}

Based on the Bondi-Sachs metric [1, 2], the characteristic or null-cone approach to numerical relativity permits a rigorous treatment of far-field gravitational radiation, including an explicit computation of the news function. This approach has been implemented in the PITT code [3-8], which is the focus of this work, as well as in a number of other numerical codes [9-15].

The eventual goal of our work is to develop a characteristic code that can reliably compute gravitational radiation in situations of astrophysical interest, either within a standalone code $[7,16]$ or within the context of Cauchy-characteristic extraction or matching 
[17]. The PITT code can stably evolve single black hole spacetimes for long times [18] and accurately compute the emitted gravitational radiation in test cases [3,5] and in scattering problems [19]. However, it cannot yet accurately compute the gravitational radiation emitted in astrophysically interesting scenarios, such as a star in close orbit around a black hole [7, 16].

In this paper, we investigate a strategy for improving the PITT code's numerical accuracy. Up till now, the PITT code has used second-order finite differencing, with two stereographic (angular) coordinate patches, one each covering the northern and southern hemispheres. Unfortunately, numerical noise from the two-dimensional interpatch interpolation seems to be a major limitation in the code's ability to accurately compute gravitational radiation emission. Here we investigate a modification of the code to use the six-angular-patch scheme and infrastructure of [20], and a second modification to use fourth-order finite differencing in the angular directions. ${ }^{3}$

In comparison to the two-patch stereographic scheme, the six-patch scheme allows the angular coordinates to be chosen such that the interpatch interpolation is only one dimensional, leading to an expectation of reduced noise at the patch interfaces. Having the interpatch interpolation be one dimensional also makes it relatively easy to use higher order interpolation, as is appropriate to match the higher (fourth) order angular finite differencing. As well, the six-patch scheme has considerably less distortion of the finite differencing grid near the patch edges than the stereographic scheme.

There have been a number of other recent uses of multiple-patch finite differencing schemes (also known as multiple-block or multiple-domain schemes) for evolutions in numerical relativity. Reference [20] used a six-patch 'inflated-cube' scheme, with the BSSN formulation of the Einstein equations, to evolve excised Kerr black holes. [21] and [22] considered the axisymmetric evolution of a scalar field on a Schwarzschild background, and boosted Kerr background, respectively, using overlapping spherical polar and cylindrical patches. [23] used a pair of overlapping spherical polar inner patches and an outer Cartesian patch to simulate boosted Kerr black holes. In the schemes described thus far, adjacent patches overlap and state-vector values are interpolated between the patches as necessary near the interpatch boundaries. In contrast, [24-27] use a different approach, where adjacent patches just touch and ingoing/outgoing modes are transferred between the patches at the interpatch boundary points. Long-term stability and high accuracy in studies of scalar field tails in Schwarzschild or Kerr spacetime were reported.

In the null-cone formalism, as in the ADM formalism, four of the ten characteristic Einstein equations are not used in the evolution but constitute constraints. We have constructed and validated code that evaluates the constraints, so as to have a tool to monitor the reliability of a computational evolution. As test cases for the code, we use a class of exact solutions to the linearized Einstein equations [28]. These solutions are written in terms of the Bondi-Sachs metric and continuously emit gravitational radiation.

The numerical computations presented here were all performed within the Cactus computational toolkit [29] (http://www.cactuscode.org), using the Carpet driver [30] (http://www.carpetcode.org) to support the multiple-patch computations. The computer algebra results were obtained using Maple.

The plan of the paper is as follows. Section 2 summarizes background material that will be used later. Section 3 describes our implementation of the six-patch angular coordinate system. Section 4 describes the constraint evaluation. Computational results are presented in section 5, and are then discussed in the conclusion, section 6.

\footnotetext{
3 Unfortunately, the code remains globally second order because the time evolution and the radial finite differencing remain second order-these derivatives are not localized in a single subroutine, so changing them would require a major rewrite of the code.
} 


\section{Background material}

\subsection{The Bondi-Sachs metric}

The formalism for the numerical evolution of Einstein's equations, in null-cone coordinates, is well known [1, 3-6, 31]. For the sake of completeness, we give a summary of those aspects of the formalism that will be used here. We start with coordinates based upon a family of outgoing null hypersurfaces. We let $u$ label these hypersurfaces, $x^{A}(A=2,3)$, label the null rays and $r$ be a surface area coordinate. In the resulting $x^{\alpha}=\left(u, r, x^{A}\right)$ coordinates, the metric takes the Bondi-Sachs form $[1,2]$

$$
\begin{aligned}
\mathrm{d} s^{2}=-\left(\mathrm{e}^{2 \beta}(1\right. & \left.\left.+W_{c} r\right)-r^{2} h_{A B} U^{A} U^{B}\right) \mathrm{d} u^{2} \\
& -2 \mathrm{e}^{2 \beta} \mathrm{d} u \mathrm{~d} r-2 r^{2} h_{A B} U^{B} \mathrm{~d} u \mathrm{~d} x^{A}+r^{2} h_{A B} \mathrm{~d} x^{A} \mathrm{~d} x^{B},
\end{aligned}
$$

where $h^{A B} h_{B C}=\delta_{C}^{A}$ and $\operatorname{det}\left(h_{A B}\right)=\operatorname{det}\left(q_{A B}\right)$, with $q_{A B}$ being a metric representing a unit 2-sphere embedded in flat Euclidean 3-space; $W_{c}$ is a normalized variable used in the code, related to the usual Bondi-Sachs variable $V$ by $V=r+W_{c} r^{2}$. As discussed in more detail below, we represent $q_{A B}$ by means of a complex dyad $q_{A}$. Then, for an arbitrary Bondi-Sachs metric, $h_{A B}$ can then be represented by its dyad component

$$
J=h_{A B} q^{A} q^{B} / 2
$$

with the spherically symmetric case characterized by $J=0$. We also introduce the spinweighted field

$$
U=U^{A} q_{A},
$$

as well as the (complex differential) eth operators $ð$ and $\bar{\varnothing}$ [32].

Einstein's equations $R_{\alpha \beta}=8 \pi\left(T_{\alpha \beta}-\frac{1}{2} g_{\alpha \beta} T\right)$ are classified as hypersurface equations$R_{11}, q^{A} R_{1 A}, h^{A B} R_{A B}$-forming a hierarchical set for $\beta, U$ and $W_{c}$; evolution equation $q^{A} q^{B} R_{A B}$ for $J$; and constraints $R_{0 \alpha}$. An evolution problem is normally formulated in the region of spacetime between a timelike or null worldtube and future null infinity, with (free) initial data $J$ given on $u=0$, and with boundary data for $\beta, U, W_{c}, J$ satisfying the constraints given on the inner worldtube.

\subsection{The spin-weighted formalism and the $\mathrm{x}$ operator}

A complex dyad is written as

$$
q_{A}=\left(r_{2} \mathrm{e}^{\mathrm{i} \phi_{2}}, r_{3} \mathrm{e}^{\mathrm{i} \phi_{3}}\right),
$$

where $r_{A}, \phi_{A}$ are real quantities (but in general they are not vectors). The real and imaginary parts of $q_{A}$ are unit vectors that are orthogonal to each other, and $q_{A}$ represents the metric. Thus

$$
q^{A} q_{A}=0, \quad q^{A} \bar{q}_{A}=2, \quad q_{A B}=\frac{1}{2}\left(q_{A} \bar{q}_{B}+\bar{q}_{A} q_{B}\right) .
$$

It is straightforward to substitute a 2-metric into equation (5) to find $r_{A}$ and $\left(\phi_{3}-\phi_{2}\right)$. Thus $q_{A}$ is not unique, up to a unitary factor: if $q_{A}$ represents a given 2-metric, then so does $q_{A}^{\prime}=\mathrm{e}^{\mathrm{i} \alpha} q_{A}$. Thus, considerations of simplicity are used in deciding the precise form of dyad to represent a particular 2-metric. For example, the dyads commonly used to represent some unit sphere metrics, namely spherical polars and stereographic, are

$$
\begin{aligned}
& \mathrm{d} s^{2}=\mathrm{d} \theta^{2}+\sin ^{2} \theta^{2} \mathrm{~d} \phi^{2}: q_{A}=(1, \mathrm{i} \sin \theta) \\
& \mathrm{d} s^{2}=\frac{4\left(d q^{2}+d p^{2}\right)}{\left(1+q^{2}+p^{2}\right)^{2}}: q_{A}=\frac{2}{1+q^{2}+p^{2}}(1, \mathrm{i}) .
\end{aligned}
$$


Having defined a dyad, we may construct complex quantities representing all manner of tensorial objects, for example $X_{1}=T_{A} q^{A}, X_{2}=T^{A B} q_{A} \bar{q}_{B}, X_{3}=T_{C}^{A B} \bar{q}_{A} \bar{q}_{B} \bar{q}^{C}$. Each object has no free indices, and has associated with it a spin weight $s$ defined as the number of $q$ factors less the number of $\bar{q}$ factors in its definition. For example, $s\left(X_{1}\right)=1, s\left(X_{2}\right)=0, s\left(X_{3}\right)=-3$, and, in general, $s(X)=-s(\bar{X})$. We define derivative operators $\varnothing$ and $\bar{\varnothing}$ acting on a quantity $V$ with spin weight $s$

$$
\text { ð } V=q^{A} \partial_{A} V+s \Gamma V, \quad \bar{\chi} V=\bar{q}^{A} \partial_{A} V-s \bar{\Gamma} V,
$$

where the spin weights of $\chi V$ and $\bar{\partial} V$ are $s+1$ and $s-1$, respectively, and where

$$
\Gamma=-\frac{1}{2} q^{A} \bar{q}^{B} \nabla_{A} q_{B}
$$

In the case of spherical polar coordinates, $\Gamma=-\cot \theta$, and for stereographic coordinates $\Gamma=q+\mathrm{i} p$.

The spin weights of the quantities used in the Bondi-Sachs metric are

$$
s\left(W_{c}\right)=s(\beta)=0, \quad s(J)=2, \quad s(\bar{J})=-2, \quad s(U)=1, s(\bar{U})=-1 .
$$

We will be using spin-weighted spherical harmonics [33, 34] using the formalism described in [19]. It will prove convenient to use ${ }_{s} Z_{\ell m}$ rather than the usual ${ }_{s} Y_{\ell m}$ (the $\operatorname{prefix}_{s}$ denotes the spin weight) as basis functions, where

$$
\begin{aligned}
{ }_{s} Z_{\ell m} & =\frac{1}{\sqrt{2}}\left[{ }_{s} Y_{\ell m}+(-1)^{m}{ }_{s} Y_{\ell-m}\right] & \text { for } & m>0 \\
{ }_{s} Z_{\ell m} & =\frac{\mathrm{i}}{\sqrt{2}}\left[(-1)^{m}{ }_{s} Y_{\ell m}-{ }_{s} Y_{\ell-m}\right] & \text { for } & m<0 \\
{ }_{s} Z_{\ell 0} & ={ }_{s} Y_{\ell 0} . & &
\end{aligned}
$$

Note that [19] uses the notation ${ }_{s} R_{\ell m}$ rather than the ${ }_{s} Z_{\ell m}$ used here; we use a different notation to avoid any confusion with the Ricci tensor. In the case $s=0$, the $s$ will be omitted, i.e. $Z_{\ell m}={ }_{0} Z_{\ell m}$. Note that the effect of the $ð$ operator acting on $Z_{\ell m}$ is

$$
ð Z_{\ell m}=\sqrt{\ell(\ell+1)} Z_{\ell m}, \quad \partial^{2} Z_{\ell m}=\sqrt{(\ell-1) \ell(\ell+1)(\ell+2)}{ }_{2} Z_{\ell m} .
$$

\subsection{Linearized solutions}

A class of solutions, in Bondi-Sachs form, to the linearized Einstein equations in vacuum was presented in [28], and we use these solutions to test the accuracy of the numerical evolutions described later. More specifically, the solutions to be used are those given in section 4.3 of [28] for the case of a dynamic spacetime on a Minkowski background. We write

$$
\begin{aligned}
& J=\sqrt{(\ell-1) \ell(\ell+1)(\ell+2)}{ }_{2} Z_{\ell m} \operatorname{Re}\left(J_{\ell}(r) \mathrm{e}^{\mathrm{i} v u}\right), \\
& U=\sqrt{\ell(\ell+1)_{1}} Z_{\ell m} \operatorname{Re}\left(U_{\ell}(r) \mathrm{e}^{\mathrm{i} v u}\right), \\
& \beta=Z_{\ell m} \operatorname{Re}\left(\beta_{\ell} \mathrm{e}^{\mathrm{i} v u}\right), \\
& W_{c}=Z_{\ell m} \operatorname{Re}\left(W_{c \ell}(r) \mathrm{e}^{\mathrm{i} v u}\right),
\end{aligned}
$$

where $J_{\ell}(r), U_{\ell}(r), \beta_{\ell}, W_{c \ell}(r)$ are in general complex, and taking the real part leads to $\cos (\nu u)$ and $\sin (v u)$ terms. The quantities $\beta$ and $W_{c}$ are real; while $J$ and $U$ are complex due to the terms $\partial^{2} Z_{\ell m}$ and $ð Z_{\ell m}$, representing different terms in the angular part of the metric. We 
require a solution that is well behaved at future null infinity, and is well defined for $r \geqslant 2$, at which surface we set the inner boundary. We find in the case $\ell=2$ :

$\beta_{2}=\beta_{0}$,

$J_{2}(r)=\frac{24 \beta_{0}+3 \mathrm{i} \nu C_{1}-\mathrm{i} \nu^{3} C_{2}}{36}+\frac{C_{1}}{4 r}-\frac{C_{2}}{12 r^{3}}$,

$U_{2}(r)=\frac{-24 \mathrm{i} v \beta_{0}+3 v^{2} C_{1}-v^{4} C_{2}}{36}+\frac{2 \beta_{0}}{r}+\frac{C_{1}}{2 r^{2}}+\frac{\mathrm{i} \nu C_{2}}{3 r^{3}}+\frac{C_{2}}{4 r^{4}}$,

$W_{c 2}(r)=\frac{24 \mathrm{i} v \beta_{0}-3 v^{2} C_{1}+v^{4} C_{2}}{6}+\frac{3 \mathrm{i} v C_{1}-6 \beta_{0}-\mathrm{i} \nu^{3} C_{2}}{3 r}-\frac{v^{2} C_{2}}{r^{2}}+\frac{\mathrm{i} \nu C_{2}}{r^{3}}+\frac{C_{2}}{2 r^{4}}$,

with the (complex) constants $\beta_{0}, C_{1}$ and $C_{2}$ freely specifiable.

We find in the case $\ell=3$ :

$\beta_{2}=\beta_{0}$,

$J_{3}(r)=\frac{60 \beta_{0}+3 \mathrm{i} \nu C_{1}+v^{4} C_{2}}{180}+\frac{C_{1}}{10 r}-\frac{\mathrm{i} \nu C_{2}}{6 r^{3}}-\frac{C_{2}}{4 r^{4}}$,

$U_{3}(r)=\frac{-60 \mathrm{i} \nu \beta_{0}+3 v^{2} C_{1}-\mathrm{i} v^{5} C_{2}}{180}+\frac{2 \beta_{0}}{r}+\frac{C_{1}}{2 r^{2}}-\frac{2 v^{2} C_{2}}{3 r^{3}}+\frac{5 \mathrm{i} \nu C_{2}}{4 r^{4}}+\frac{C_{2}}{r^{5}}$,

$W_{c 3}(r)=\frac{60 \mathrm{i} v \beta_{0}-3 v^{2} C_{1}+\mathrm{i} v^{5} C_{2}}{15}+\frac{\mathrm{i} \nu C_{1}-2 \beta_{0}+v^{4} C_{2}}{3 r}$

$$
-\frac{\mathrm{i} 2 v^{3} C_{2}}{r^{2}}-\frac{4 \mathrm{i} v^{2} C_{2}}{r^{3}}+\frac{5 v C_{2}}{r^{4}}+\frac{3 C_{2}}{r^{5}}
$$

The emitted gravitational radiation, that is the news $N$, takes a simple form in the linearized limit when the metric satisfies equation (12):

$N=\operatorname{Re}\left(\mathrm{e}^{\mathrm{i} v u} \lim _{r \rightarrow \infty}\left(\frac{\ell(\ell+1)}{4} J_{\ell}-\frac{\mathrm{i} v}{2} r^{2} J_{\ell, r}\right)+\mathrm{e}^{\mathrm{i} v u} \beta_{\ell}\right) \sqrt{(\ell-1) \ell(\ell+1)(\ell+2)_{2}} Z_{\ell m}$.

For the cases $\ell=2$ and 3 ,

$\ell=2: N=\operatorname{Re}\left(\frac{\mathrm{i} v^{3} C_{2}}{\sqrt{24}} \mathrm{e}^{\mathrm{i} v u}\right)_{2} Z_{2 m} ; \quad \ell=3: N=\operatorname{Re}\left(\frac{-v^{4} C_{2}}{\sqrt{30}} \mathrm{e}^{\mathrm{i} v u}\right)_{2} Z_{3 m}$.

\subsection{Six-patch angular coordinates}

Given Cartesian coordinates $(x, y, z)$, we define 'inflated-cube' angular coordinates on each 2-sphere of constant $u$ and $r$,

$$
\begin{aligned}
& \mu \equiv \text { rotation angle about the } x \text { axis }=\arctan (y / z) \\
& \nu \equiv \text { rotation angle about the } y \text { axis }=\arctan (x / z) \\
& \phi \equiv \text { rotation angle about the } z \text { axis }=\arctan (y / x),
\end{aligned}
$$

where all the arctangents are 4-quadrant based on the signs of $x, y$ and $z$. We then introduce six coordinate patches covering neighbourhoods of the $\pm z, \pm x$ and $\pm y$ axes, with the angular coordinates $x^{A} \equiv(\rho, \sigma)$ in each patch defined as follows (see note $\left.{ }^{4}\right)$ :

$$
(\rho, \sigma) \equiv \begin{cases}(\nu, \mu) & \text { in } \pm z \text { patches } \\ (\nu, \phi) & \text { in } \pm x \text { patches } \\ (\mu, \phi) & \text { in } \pm y \text { patches. }\end{cases}
$$
4 This definition differs from that of [20] only by the interchange of $\rho$ and $\sigma$ in $\pm z$ patches. The present definition
has the advantage of having a consistent parity, with $\vec{\rho} \times \vec{\sigma}$ always pointing \{outwards, inwards\} in $\{+,-\}$ patches. 


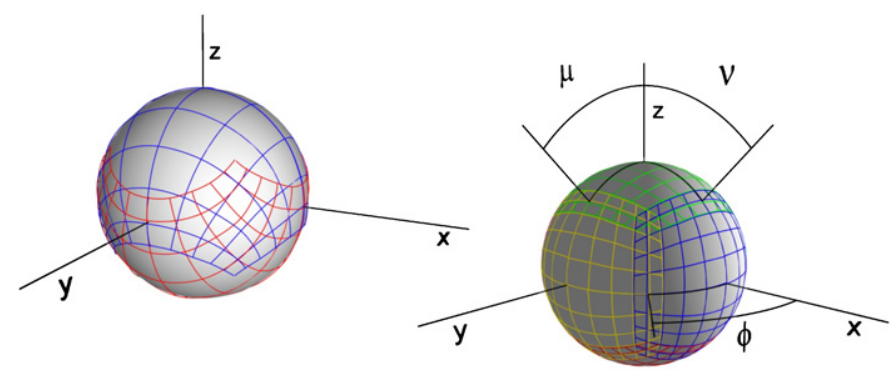

Figure 1. These figures show stereographic (left) and six-patch mapping (right) covering $S^{2}$. Large distortions in the overlap region of the stereographic mapping may cause inaccuracies. Furthermore, 2D interpolation is necessary. Six patches on the other hand reduce the distortions and only $1 \mathrm{D}$ interpolation is necessary.

Note that each patch's $x^{A}$ coordinates are nonsingular throughout a neighbourhood of the patch, and that the union of all the patches covers $S^{2}$ without coordinate singularities. The name 'inflated cube' comes from another way to visualize these patches and coordinates: imagine an $x y z$ cube with $x y z$ grid lines painted on its faces. Now imagine the cube to be flexible, and inflate it like a balloon, so it becomes spherical in shape. The resulting coordinate lines will closely resemble those for $(\mu, v, \phi)$ coordinates.

We introduce ghost zones in the usual manner along the angular boundaries of each patch, and we refer to the non-ghost-zone part of each patch's numerical grid as the 'nominal' grid. We size the patches so they overlap slightly, with each ghost-zone grid point lying in the nominal grid of some other patch. Figure 1 shows an example of a six-patch system of this type.

We couple the patches together by interpolating the field variables from neighbouring patches to each ghost-zone grid point. Note that with the definition (18), the angular coordinate $x^{A}$ perpendicular to an interpatch boundary is always common to both the adjacent patches. This means that the interpatch interpolation need only be done in one dimension, parallel to the interpatch boundary.

Each patch uses its own $x^{A} \equiv(\rho, \sigma)$ dyad for the spin-weighted quantities; when interpolating data from one patch to another we transform the data (after interpolation) as described in section 3.1 .

\section{Implementation of six-patch angular coordinates}

\subsection{Spin-weighted formalism}

The unit sphere metric $q_{A B}$ in each patch is written as

$\mathrm{d} s^{2}=\left(1-\sin ^{2} \rho \sin ^{2} \sigma\right)^{-2}\left(\cos ^{2} \sigma \mathrm{d} \rho^{2}+\cos ^{2} \rho \mathrm{d} \sigma^{2}-\frac{1}{2} \sin (2 \rho) \sin (2 \sigma) \mathrm{d} \rho \mathrm{d} \sigma\right)$,

with respect to coordinates $(\rho, \sigma)$ with range $(-\pi / 4,+\pi / 4) \times(-\pi / 4,+\pi / 4)$. A (simple) dyad representing equation (19) is

$q_{A}=\left(\frac{\left(\theta_{c}+\mathrm{i} \theta_{s}\right) \cos \sigma}{4 \theta_{c}^{2} \theta_{s}^{2}}, \frac{\left(\theta_{c}-\mathrm{i} \theta_{s}\right) \cos \rho}{4 \theta_{c}^{2} \theta_{s}^{2}}\right), \quad q^{A}=\left(2 \theta_{c} \theta_{s} \frac{\theta_{s}+\mathrm{i} \theta_{c}}{\cos \sigma}, 2 \theta_{c} \theta_{s} \frac{\theta_{s}-\mathrm{i} \theta_{c}}{\cos \rho}\right)$,

where

$$
\theta_{c}=\sqrt{\frac{1-\sin \rho \sin \sigma}{2}}, \quad \theta_{s}=\sqrt{\frac{1+\sin \rho \sin \sigma}{2}} .
$$


The factor $\Gamma$, defined in equation (8) and needed for the evaluation of $\varnothing$, works out to be

$$
\begin{aligned}
\Gamma=\frac{\cos ^{2} \rho \cos ^{2} \sigma(\sin \rho+\sin \sigma)+\left(\cos ^{2} \rho-\cos ^{2} \sigma\right)(\sin \sigma-\sin \rho)}{4 \theta_{c} \cos \sigma \cos \rho} \\
+i \frac{\cos ^{2} \rho \cos ^{2} \sigma(\sin \rho-\sin \sigma)+\left(\cos ^{2} \sigma-\cos ^{2} \rho\right)(\sin \rho+\sin \sigma)}{4 \theta_{s} \cos \sigma \cos \rho} .
\end{aligned}
$$

In addition, we need to specify how spin-weighted quantities transform at interpatch boundaries. Suppose that we have two patches 'old' and 'new', with quantities in the two patches being denoted by means of suffixes $(O)$ and $(N)$, respectively. Define the Jacobian $J_{B}^{A}$ from the old patch to the new patch

$$
J_{B}^{A}=\frac{\partial x_{(N)}^{A}}{\partial x_{(O)}^{B}} .
$$

Then the dyad $q_{(O)}^{A}$ has components in the new coordinates

$$
q_{(O)[N]}^{A}=q_{(O)}^{B} J_{B}^{A},
$$

where the notation is that the $(O)$ indicates that we are referring to the dyad that generates the metric in the old patch, and the ${ }_{[N]}$ indicates that the components are given with respect to the new coordinates. Any two complex dyads are related by means of a rotation exp(i $\gamma)$. Writing

$$
q_{(N)}^{A}=\exp (\mathrm{i} \gamma) q_{(O)[N]}^{A},
$$

and applying equation (5), it follows that

$$
\exp (\mathrm{i} \gamma)=\frac{2}{q_{A B(N)} \bar{q}_{(N)}^{A} q_{(O)[N]}^{B}},
$$

and then a spin-weighted quantity $V$ with spin weight $s$ transforms between the two patches as

$$
V_{(N)}=\exp (\mathrm{is} \gamma) V_{(O)} \text {. }
$$

There are many different cases for the transformation between the patches, and we give the transformation and Jacobian explicitly in only one case, when the interpatch boundary is at

$$
\rho_{(O)}=\frac{\pi}{4}, \quad \rho_{(N)}=-\frac{\pi}{4}, \quad \sigma_{(O)}=\sigma_{(N)} .
$$

Then the coordinate transformation is

$$
\rho_{(N)}=\arctan \left(-\frac{1}{\tan \rho_{(O)}}\right), \quad \sigma_{(N)}=\arctan \left(\frac{\tan \sigma_{(O)}}{\tan \rho_{(O)}}\right),
$$

and the Jacobian evaluates to

$$
\begin{aligned}
& \frac{\partial \rho_{(N)}}{\partial \rho_{(O)}}=1, \quad \frac{\partial \rho_{(N)}}{\partial \sigma_{(O)}}=0, \\
& \frac{\partial \sigma_{(N)}}{\partial \rho_{(O)}}=-\frac{\cos \sigma \sin \sigma}{\cos ^{2} \sigma \sin ^{2} \rho+\cos ^{2} \rho \sin ^{2} \sigma}, \quad \frac{\partial \sigma_{(N)}}{\partial \sigma_{(O)}}=\frac{\cos \rho \sin \rho}{\cos ^{2} \sigma \sin ^{2} \rho+\cos ^{2} \rho \sin ^{2} \sigma} .
\end{aligned}
$$




\subsection{Computational implementation}

The existing stereographic code has been extended to the six-patch coordinate system. Since the formulation of the equations in terms of spin-weighted quantities is independent of angular coordinate bases but dyad dependent, it is necessary to reimplement only those objects that depend on the six-patch dyad. We have therefore provided a numerical implementation of the new $\partial$-operators in the code. In addition, we have adapted the spin-transformation coefficients to the six patches. In order to test the six-patch code against the linearized solutions, the spin-weighted spherical harmonics ${ }_{s} Z_{\ell m}$ needed to be implemented for the six-patch coordinates and dyad.

The ठ-operators are implemented via subroutines $D_{1}(s, e)$ and $D_{2}\left(s, e_{1}, e_{2}\right)$, which

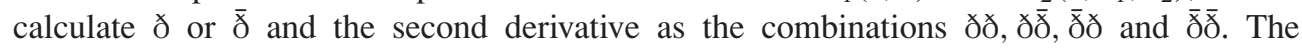
parameter $s$ specifies the spin $s$ of the quantity to which the operator is applied, and $e_{1}, e_{2} \in\{-1,1\}$ denote $\chi$ and $\bar{\chi}$.

The derivative operators $\partial_{\rho}^{2}, \partial_{\sigma}^{2}, \partial_{\rho} \partial_{\sigma}, \partial_{\rho}$ and $\partial_{\sigma}$ have been approximated by second- and fourth-order accurate centred finite difference stencils [35]. The six-patch code uses either cubic or quintic Lagrange polynomial interpolation between the patches, correspondingly. After the interpolation, we apply the transformation law (27) to transform quantities of spin weight $s \neq 0$ to the current patch's coordinates and dyad.

It turns out that we need a total number of 12 different spin-transformation coefficients, since we have a $P_{ \pm}$-symmetry between the total number of 24 ghost zones across all $P_{+i}$ and $P_{-i}, i=x, y, z$ patches. These coefficients are calculated and stored for repeated use in an initial routine. After each radial step during the integration of the characteristic equations, the ghost zones are synchronized by the multipatch infrastructure, and afterwards, the code multiplies the appropriate spin-transformation coefficients with the synchronized values of the ghost zones.

We have carried out checks of the angular grid including the $D_{1}$ - and $D_{2}$-operators and the spin-transformation coefficients, and we have found out that the code converges with secondand fourth-order accuracy, respectively.

The spin-weighted spherical harmonics have been implemented by using the spherical harmonics in terms of the stereographic coordinate $\zeta=p+\mathrm{i} q$ and by applying the pseudonumerical operators $D_{1}$ and $D_{2}$ in order to obtain ${ }_{s} Z_{\ell m}$ for $|s|>0$. (By 'pseudo-numerical', we mean that we apply the fourth-order $D_{1}$ and $D_{2}$ operators with a very small delta spacing such that we reach machine precision, since we know the $Z_{\ell m}$ everywhere and are not bound to the numerical grid.) In order to use the stereographic routines for the $Z_{\ell m}$, we have transformed the stereographic coordinate $\zeta$ to the six-patch coordinates and depending on the hemisphere, we use the stereographic routines for north or south patch, respectively.

Furthermore, we have implemented an algorithm for calculating the linearized news function (equation (15)), in the form

$$
N=\lim _{r \rightarrow \infty}\left(\frac{\ell(\ell+1)}{4} J-\frac{1}{2} r^{2} J_{, u r}+\partial^{2} \beta\right) .
$$

\section{The constraint equations}

If the boundary data satisfy the constraints $R_{0 \alpha}=0$ (here we restrict attention to the vacuum case), and provided the hypersurface and evolution equations are satisfied, the Bianchi identities guarantee that the constraints are satisfied throughout the domain $[1,2]$. Thus from an analytic viewpoint, evaluation of the constraints is redundant, but in a numerical 
simulation their evaluation may provide useful information concerning the reliability of the computation.

We have written the code that uses the Bondi-Sachs metric variables and derivatives to evaluate $R_{00}, R_{01}$ and $q^{A} R_{0 A}$. The expressions for these quantities are very long and are not reproduced here; instead, they are available online as a multimedia supplement to this paper (available at stacks.iop.org/CQG/24/S327). The Fortran code for the expressions was generated directly from the computer algebra (Maple) output.

\section{Testing the code}

In this section we first specify the linearized solutions against which the code will be tested, as well as the various parameters that describe a numerical solution and its output. Then we present the results of testing the comparative performance of the second-order six patch, fourth-order six patch and stereographic codes.

We refer to the linearized solutions summarized in section 2.3. In all cases we take $v=1$ and $m=0$. We present results for the cases $\ell=2$ and $\ell=3$ with

$$
\begin{aligned}
& C_{1}=3 \times 10^{-6}, \quad C_{2}=10^{-6}, \quad \beta_{0}=i \times 10^{-6} \quad(\ell=2) \\
& C_{1}=3 \times 10^{-6}, \quad C_{2}=i \times 10^{-6}, \quad \beta_{0}=i \times 10^{-6} \quad(\ell=3)
\end{aligned}
$$

in equation (13) in the case $\ell=2$, and in equation (14) in the case $\ell=3$.

All the numerical simulations use a compactified radial coordinate $x=r /\left(r_{w t}+r\right)$ with $r_{w t}=9$. Data are prescribed at time $u=0$ as well as at the inner boundary $r=2$ (which is equivalent to $x=0.1888$ ). The stereographic grids (with ghost zones excluded) are

$$
\text { coarse: } n_{x}=n_{q}=n_{p}=41, \quad \text { fine: } n_{x}=n_{q}=n_{p}=81,
$$

and there is no overlap between the two patches, i.e. we set the code parameter $q_{\text {size }}=1$ which means that on the nominal grid, the holomorphic coordinate function $\zeta=q+\mathrm{i} p$ takes values in $q, p \in[-1,1]$. The six-patch grids are such that, over the whole sphere, the total number of angular cells is equivalent. We take

coarse: $n_{x}=41, \quad n_{\sigma}=n_{\rho}=24, \quad$ fine: $n_{x}=81, \quad n_{\sigma}=n_{\rho}=47$.

Six-patch results are reported for both second-order and fourth-order differencing of the angular derivatives. In all cases, the fine grid has $\Delta u=0.0125$ and the coarse grid has $\Delta u=0.025$. Runs are performed for two complete periods, i.e. starting at $u=0$ and ending at $u=4 \pi$. Results are reported for the errors of the quantities shown using the $L_{2}$ (rootmean-square) norm, evaluated at the time shown, averaged over all non-ghost grid-points over the whole sphere and between the inner boundary and future null infinity. The norm of the error in the news is averaged over the whole sphere at future null infinity.

Given a quantity $\Psi$, the error $\varepsilon$ and the associated convergence factor $C$ are defined as

$$
\varepsilon=\left\|\Psi_{\text {numeric }}-\Psi_{\text {analytic }}\right\|, \quad C=\frac{\varepsilon_{\text {coarse }}}{\varepsilon_{\text {fine }}} .
$$

Thus [36], $C=2$ corresponds to first-order, and $C=4$ to second-order convergence. As discussed in the introduction, we expect the code to exhibit second-order convergence $(C=4)$ in the limit of infinite resolution, even when using fourth-order accurate angular derivatives.

Figure 2 shows the error norms $\varepsilon(t)$ for the metric quantity $J$, and for the Bondi news $N$, plotted against time in the case $\ell=2$. Each panel of the figure plots $\varepsilon(t)$ for the three schemes stereographic, six-patch second order, and six-patch fourth order; and in each case, we plot $4 \cdot \varepsilon(t) / 4$ at fine resolution (points), and $\varepsilon(t)$ at coarse resolution (solid line), so that 
Table 1. This table shows the error norm $\varepsilon$ for the low resolution runs, and the convergence factor $C$, both averaged over space and time, for each version of the code and for each of five diagnostic quantities: the metric variable $J$, the news function $N$, and the constraints $R_{00}, R_{01}$ and $R_{0 A}$.

\begin{tabular}{|c|c|c|c|}
\hline Quantity & Stereographic & $\begin{array}{l}\ell=\mathbf{2} \text { test data } \\
\text { Six-patch, second order }\end{array}$ & Six-patch, fourth order \\
\hline$C(J)$ & 3.8456 & 3.8286 & 3.9112 \\
\hline$\varepsilon(J)$ & $3.3039 \times 10^{-9}$ & $1.5491 \times 10^{-9}$ & $6.9157 \times 10^{-11}$ \\
\hline$C(N)$ & 3.3119 & 3.9642 & 3.5528 \\
\hline$\varepsilon(N)$ & $2.2785 \times 10^{-8}$ & $1.0913 \times 10^{-8}$ & $8.4414 \times 10^{-10}$ \\
\hline$C\left(R_{00}\right)$ & 1.2487 & 1.5000 & 2.0319 \\
\hline$\varepsilon\left(R_{00}\right)$ & $3.1942 \times 10^{-9}$ & $2.8779 \times 10^{-9}$ & $5.7668 \times 10^{-10}$ \\
\hline$C\left(R_{01}\right)$ & 3.5560 & 3.5936 & 3.1296 \\
\hline$\varepsilon\left(R_{01}\right)$ & $3.9214 \times 10^{-11}$ & $1.6988 \times 10^{-11}$ & $2.7331 \times 10^{-12}$ \\
\hline$C\left(R_{0 A}\right)$ & 3.4285 & 1.7558 & 2.0043 \\
\hline$\varepsilon\left(R_{0 A}\right)$ & $5.2549 \times 10^{-9}$ & $6.6397 \times 10^{-9}$ & $2.1543 \times 10^{-9}$ \\
\hline \multicolumn{4}{|c|}{$\ell=3$ test data } \\
\hline$C(J)$ & 3.9783 & 3.9106 & 4.0777 \\
\hline$\varepsilon(J)$ & $4.6461 \times 10^{-9}$ & $3.2784 \times 10^{-9}$ & $1.3677 \times 10^{-10}$ \\
\hline$C(N)$ & 2.1201 & 3.9134 & 3.6262 \\
\hline$\varepsilon(N)$ & $4.9174 \times 10^{-8}$ & $2.8182 \times 10^{-8}$ & $1.7996 \times 10^{-9}$ \\
\hline$C\left(R_{00}\right)$ & 1.2743 & 1.7963 & 2.0330 \\
\hline$\varepsilon\left(R_{00}\right)$ & $7.2594 \times 10^{-9}$ & $4.5824 \times 10^{-9}$ & $1.1744 \times 10^{-9}$ \\
\hline$C\left(R_{01}\right)$ & 3.5144 & 3.5383 & 3.3824 \\
\hline$\varepsilon\left(R_{01}\right)$ & $1.3262 \times 10^{-10}$ & $7.5501 \times 10^{-11}$ & $6.0924 \times 10^{-12}$ \\
\hline$C\left(R_{0 A}\right)$ & 3.4326 & 1.9510 & 2.0156 \\
\hline$\varepsilon\left(R_{0 A}\right)$ & $9.0299 \times 10^{-9}$ & $1.0076 \times 10^{-8}$ & $2.9654 \times 10^{-9}$ \\
\hline
\end{tabular}

for second-order convergence the points and solid line coincide. Table 1 gives the error norm $\varepsilon$ (coarse resolution) and the convergence rate $C$, in both cases averaged over the whole run, for the quantities indicated.

It is clear that all schemes give (approximate) second-order convergence of the metric quantity $J$. However, the news and the constraints (all of which contain second derivatives of metric quantities) exhibited, in some cases, degradation of the order of convergence. Referring to the plot of the news $N$ in figure 2, we see that the six-patch schemes were approximately second-order convergent throughout the run, but that the stereographic scheme exhibited convergence degradation that is periodic in time (Although not shown in the figure, the convergence rate is at all times better than first order). The analytic solution has period $2 \pi$, and it is interesting that degradation peaks when the analytic solution is zero. We should also mention that this type of behaviour has shown up in previous performed runs of the stereographic code and may be related to high-frequency error modes coming from angular patch interfaces or corners.

The convergence rates of the constraints appear problematic. However, the fact that the convergence rates of $R_{0 A}$ (stereographic case) and of $R_{01}$ (all cases) are nearly second order is important. This implies that the problem is not due either to (a) a simple mis-coding of the finite difference representation of derivatives, or to (b) the expressions generated by the computer algebra. This issue will require further investigation in order to be able to use constraint violation as a reliable indicator of code accuracy.

The comparative behaviour of the error norm is particularly interesting. On average, the error norm of second-order six patch was smaller than that of stereographic by a factor of 
(a)

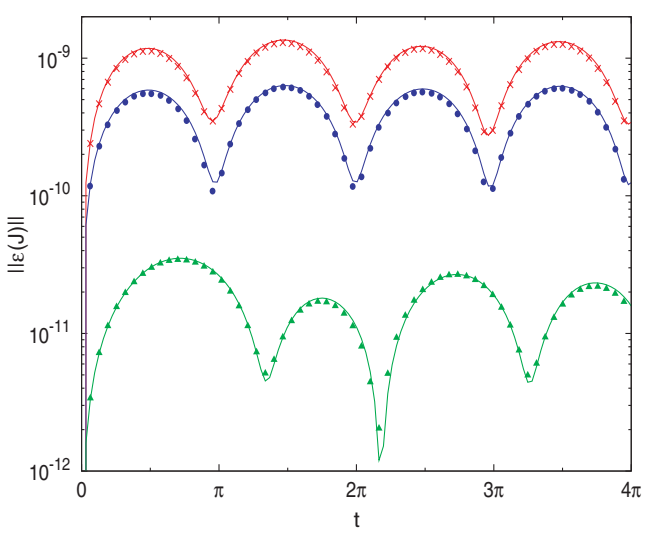

(b)

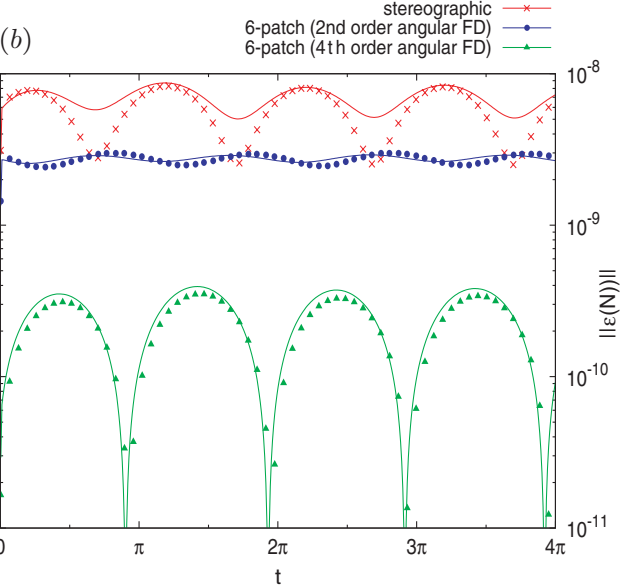

Figure 2. This figure shows an example of the convergence and accuracy of the various versions of the code. Part $(a)$ shows results for the metric variable $J$, while part $(b)$ shows results for the news function $N$; the same key applies to both parts. In all cases, the solid line shows the coarse resolution results, while the points show the fine resolution results multiplied by 4 .

order 2 (although there were cases in which the error was slightly larger). However, the fourth-order six-patch scheme exhibited a dramatic reduction in the error norm, by a factor of up to 47 compared to that of the stereographic case.

Although we used the full nonlinear version of the code, we were not able to test nonlinear effects because the analytic solution against which we measure the error is valid only in a linearized regime. Even so, it is expected that the improvement in accuracy associated with the six-patch fourth-order scheme, carries over to the nonlinear regime.

The PITT null code in the stereographic version is also capable of evolving non-vacuum spacetimes. Here, we have implemented and tested the six-patch code only for the case of vacuum. Since the ghost-zone scheme used in the six-patch system is known to be able to handle hydrodynamical shocks [37], we expect no problems in extending this method to non-vacuum spacetimes.

\section{Conclusion}

We have implemented a version of the characteristic numerical relativity code that coordinatises the sphere by means of six angular patches. Further, the six-patch code has been implemented for both second-order and fourth-order accurate finite differencing of angular derivatives. We compared the errors and rates of convergence in the various versions of the code, using exact solutions of the linearized Einstein equations as a testbed. This was done for the cases $\ell=2$ and $\ell=3$, and with several different indicators. In general, the error norm of second-order six patch was smaller than that of stereographic; and the error norm of the fourth-order six-patch scheme was much smaller, by a factor of up to 47, compared to that of the stereographic case.

Thus, we expect the six-patch characteristic code, in particular the version that uses fourth-order accurate angular finite differencing, to give significantly better performance than the stereographic version. 


\section{Acknowledgments}

NTB and CWL thank Max-Planck-Institut für Gravitationsphysik, Albert-Einstein-Institut, for hospitality; and BS and CR thank the University of South Africa for hospitality. The work was supported in part by the National Research Foundation, South Africa, under Grant number 2053724, and by SFB-TR7 'Gravitationswellenastronomie' of the German DFG. JT thanks the Alexander von Humboldt foundation for financial support.

\section{References}

[1] Bondi H, van der Burg M G J and Metzner A W K 1962 Gravitational waves in general relativity VII. Waves from axi-symmetric isolated systems Proc. R. Soc. A 269 21-52

[2] Sachs R K 1962 Gravitational waves in general relativity VIII. Waves in asymptotically flat space-time Proc. $R$. Soc. A 270 103-26

[3] Bishop N T, Gómez R, Lehner L, Maharaj M and Winicour J 1997 High-powered gravitational news Phys. Rev. D $566298-309$

[4] Gómez R 2001 Gravitational waveforms with controlled accuracy Phys. Rev. D 64024007

[5] Bishop N T, Gómez R, Lehner L and Winicour J 1996 Cauchy-characteristic extraction in numerical relativity Phys. Rev. D 54 6153-65

[6] Bishop N T, Gómez R, Lehner L, Maharaj M and Winicour J 1999 Incorporation of matter into characteristic numerical relativity Phys. Rev. D 60024005

[7] Bishop N T, Gómez R, Husa S, Lehner L and Winicour J 2003 A numerical relativistic model of a massive particle in orbit near a Schwarzschild black hole Phys. Rev. D 68084015

[8] Gómez R, Husa S, Lehner L and Winicour J 2002 Gravitational waves from a fissioning white hole Phys. Rev. D 66064019

[9] Bishop N T, Clarke C and d'Inverno R 1990 Numerical relativity on a transputer array Class. Quantum Grav. 7 L23-7

[10] dInverno R A and Vickers J A 1997 Combining Cauchy and characteristic codes: IV. The characteristic field equations in axial symmetry Phys. Rev. D 56 772-84

[11] dInverno R A, Dubal M R and Sarkies E A 2000 Cauchy-characteristic matching for a family of cylindrical solutions possessing both gravitational degrees of freedom Class. Quantum Grav. 17 3157-70

[12] Papadopoulos P and Font J A 1999 Relativistic hydrodynamics on spacelike and null surfaces: formalism and computations of spherically symmetric spacetimes Phys. Rev. D 61024015

[13] Siebel F, Font J A, Mueller E and Papadopoulos P 2003 Axisymmetric core collapse simulations using characteristic numerical relativity Phys. Rev. D 67124018

[14] Siebel F and Font J A 2001 Scalar field induced oscillations of relativistic stars and gravitational collapse Phys. Rev. D 65024021

[15] Bartnik R A and Norton A H 1999 Einstein equations in the null quasi-spherical gauge: III. Numerical algorithms Preprint gr-qc/9904045

[16] Bishop N T, Gómez R, Lehner L, Maharaj M and Winicour J 2005 Characteristic initial data for a star orbiting a black hole Phys. Rev. D 72024002

[17] Babiuc M, Szilagyi B, Hawke I and Zlochower Y 2005 Gravitational wave extraction based on Cauchycharacteristic extraction and characteristic evolution Class. Quantum Grav. 22 5089-108

[18] Gómez R, Lehner L, Marsa R L and Winicour J 1998 Moving black holes in 3D Phys. Rev. D 57 4778-88

[19] Zlochower Y, Gómez R, Husa S, Lehner L and Winicour J 2003 Mode coupling in the nonlinear response of black holes Phys. Rev. D 68084014

[20] Thornburg J 2004 Black hole excision with multiple grid patches Class. Quantum Grav. 21 3665-91

[21] Calabrese G and Neilsen D 2004 Spherical excision for moving black holes and summation by parts for axisymmetric systems Phys. Rev. D 69044020

[22] Calabrese G and Neilsen D 2005 Excising a boosted rotating black hole with overlapping grids Phys. Rev. D 71124027

[23] Anderson M W 2004 Constrained evolution in numerical relativity PhD Thesis, The University of Texas at Austin, Austin, Texas, USA

[24] Lehner L, Reula O and Tiglio M 2005 Multi-block simulations in general relativity: high order discretizations, numerical stability, and applications Class. Quantum Grav. 22 S283-322 
[25] Diener P, Dorband E N, Schnetter E and Tiglio M 2005 New, efficient, and accurate high order derivative and dissipation operators satisfying summation by parts, and applications in three-dimensional multi-block evolutions Preprint gr-qc/0512001

[26] Schnetter E, Diener P, Dorband N and Tiglio M 2006 A multi-block infrastructure for three-dimensional time-dependent numerical relativity Class. Quantum Grav. 23 S553-78

[27] Dorband E N, Berti E, Diener P, Schnetter E and Tiglio M 2006 A numerical study of the quasinormal mode excitation of Kerr black holes Phys. Rev. D 74084028

[28] Bishop N T 2005 Linearized solutions of the Einstein equations within a Bondi-Sachs framework, and implications for boundary conditions in numerical simulations Class. Quantum Grav. 22 2393-406

[29] Goodale T, Allen G, Lanfermann G, Massó J, Radke T, Seidel E and Shalf J 2003 The Cactus framework and toolkit: design and applications VECPAR'2002: Vector and Parallel Processing -5th Int. Conf. (Lecture Notes in Computer Science) (Berlin: Springer)

[30] Schnetter E, Hawley S H and Hawke I 2004 Evolutions in 3D numerical relativity using fixed mesh refinement Class. Quantum Grav. 21 1465-88

[31] Isaacson R, Welling J and Winicour J 1983 Null cone computation of gravitational radiation J. Math. Phys. 241824

[32] Gómez R, Lehner L, Papadopoulos P and Winicour J 1997 The eth formalism in numerical relativity Class. Quantum Grav. 14 977-90

[33] Newman E T and Penrose R 1966 Note on the Bondi-Metzner-Sachs group J. Math. Phys. $7863-70$

[34] Goldberg J N, MacFarlane A J, Newman E T, Rohrlich F and Sudarshan E C G 1967 Spin- $s$ spherical harmonics and ð J. Math. Phys. 8 2155-61

[35] Fornberg B 1988 Generation of finite difference formulas on arbitrarily spaced grids Math. Comput. 51 699-706

[36] Choptuik M W 1991 Consistency of finite-difference solutions to Einstein's equations Phys. Rev. D 44 3124-35

[37] Hawke I 2005 personal communication to Jonathan Thornburg 